\title{
Assisted-Leach (A-Leach) Energy Efficient Routing Protocol for Wireless Sensor Networks
}

\author{
Sunkara Vinodh Kumar and Ajit Pal
}

\begin{abstract}
The nodes in a wireless sensor network have limited energy and hence, lifetime maximization is the prime task during protocol design. Low Energy Adaptive Clustering Hierarchy (LEACH) protocol is a benchmark Clustering Protocol which imposes upon cluster heads the complete load of aggregation and relay of messages to base-station. Our protocol Assisted LEACH (A-LEACH) achieves lessened and uniform distribution of dissipated energy by separating the tasks of Routing and Data Aggregation. It introduces the concept of Helper Nodes which assist Cluster Heads for Multi-hop Routing A new algorithm has been formulated to facilitate energy efficient Multi-hop Route Setup for helper nodes to reach base station. The proposed protocol extends the lifetime of the network, minimizes overall energy dissipation in the network and distributes dissipation among Cluster Heads, Sensor Nodes and Helper Nodes vis-'a-vis LEACH. This is substantiated by simulation results.
\end{abstract}

Index Terms-Clustering protocols, energy-efficient protocol, leach, multi-hop, routing protocols.

\section{INTRODUCTION}

There have been profound studies on Wireless Sensor Networks [1] during the recent years. As the sensors in the network have limited battery power, enhancing the lifetime of a network is the basic aim of designing an energy efficient routing protocol. Clustering protocols aim to achieve energy efficiency. The whole network is divided into clusters with a cluster head node for each cluster. The data from sensors inside a cluster is aggregated at cluster head. This eliminates a lot the redundancy in packet forwarding. Low Energy Adaptive Clustering Hierarchy (LEACH) [2] is considered to be benchmark protocol in Clustering/Hierarchical based protocols [3]. Multihop-LEACH [4], LEACH-MF [5], MR-LEACH [6], and Secure LEACH [7], or M-LEACH are examples of few Clustering Protocols derived from LEACH.

In this paper, we propose Assisted-Leach Protocol abbreviated as A-LEACH. In most of the clustering protocols the whole load of data aggregation and data routing is done by cluster heads. LEACH protocol [2] directly transmits aggregated data from cluster heads to the base station. This debilitate the lifetime of a network. We introduce the concept of Helper Nodes where a node closer to the base station in every cluster is assigned the routing job whereas cluster heads take care of data aggregation. We formulate a new idea for route formulation for the helper nodes to reach base station. Every helper node chooses as the next hop, the node nearest to the base station from all its neighboring helper

Manuscript received February 10, 2013; revise April 16, 2013.

The authors are with the Computer Science and Engineering Department (e-mail: vk.86.811@gmail.com, apal@cse.iitkgp.ernet.in). nodes. We use the Receive Signal Strength of Base Station Beckon signals to decide upon nodes nearer to base station in Helper nodes selection and route set up phases. Thus the dissipation energy is lessened due to multi-hop routing and the same is distributed among helper and cluster head nodes. We propose algorithms for helper node selection and multi-hop routing. Our algorithm for cluster head selection is an extension to LEACH's cluster head selection.

\section{ALGORITHM FOR ASSISTED-LEACH}

Assisted Leach protocol has the following sub-stages:

- Cluster Head Selection

- Cluster Formation

- Helper Node Selection

- Routing Set-Up

- Sensing, Aggregating and Routing

\section{A. Cluster Head Selection}

The Cluster Head selection follows an extended procedure to Leach's [7] Cluster Head Selection. Each Node calculates its threshold based on the Formula:

$$
T(n)=\left\{\begin{array}{cl}
\frac{P}{1-P \times\left(r \bmod \left\lfloor\frac{1}{P}\right\rfloor\right)} & \text { if } n \in G \\
0.5 \times \frac{P}{1-P \times\left(r \bmod \left\lfloor\frac{1}{P}\right\rfloor\right)} & \text { if } n \in H \\
0 & \text { otherwise }
\end{array}\right.
$$

where

$P$ : Desired Percentage of Cluster Heads

$r$ : Current Round in protocol operation

$G$ : Set of Nodes that have neither been Cluster Heads nor been Helper Nodes in the last $\left\lfloor\frac{1}{P}\right\rfloor$ Rounds.

$H$ : Set of Nodes that haven't been Cluster Heads but played role of Helper Nodes in the last $\left\lfloor\frac{1}{P}\right\rfloor$ Rounds

Each sensor elects itself to be a Cluster Head by picking up a random number between '0' and '1' and comparing it to be less than the threshold.

\section{B. Cluster Formation}

Cluster Heads broadcast a HEAD_BOAST message containing their IDs to facilitate cluster formation. It can happen that a non-cluster node receives such messages from different Cluster Heads. They decide upon the Cluster Head whose message possesses highest Received Signal Strength to be their head and send a JOIN_CLUSTER packet with their IDs to corresponding Cluster Heads showing consent to be part of their clusters. 


\section{Helper Node Selection}

- Helper Node in a Cluster is the node which is nearer to the base station with sufficient remaining energy

- Base Station sends a packet containing its ID to every node assuming that the base station can reach every node at single hop over a common channel

- The nodes in each cluster store the Base Station ID from the received packet and then make a packet "RSS_PACKET" with the Received Signal Strength values (RSS Values) and (Self) Node ID as entries

- A copy of this "RSS_PACKET" is sent to the corresponding Cluster Heads

- Cluster Head elects the node which possessed packet with highest Received Signal Strength to be the Helper Node and sends an acknowledgement packet "Helper_BOAST" of the same to the respective node

- In case of a tie with few nodes possessing same RSS values for the packets from base station, Cluster Head selects the one with highest remaining energy as the Helper Node

- This way, every Cluster possesses a Cluster Head and Helper Node by now

D. Routing Set-Up

- This stage aims at finding the helper node at next hop for each helper node to route aggregated data to base station

- In this stage, only the helper nodes are operational and all other nodes including cluster heads go into sleep mode

- Each Helper Node sends the "RSS_PACKET" made in Helper Nodes Selection Phase to nodes in transmission range

- Thereby, every helper node receives "RSS_PACKET" from all its neighbours

- According to Helper Nodes Selection phase above, "RSS_PACKET" contains the Received Signal Strength (RSS) values of the packets received by corresponding neighbouring helper nodes from the base station as first entry and corresponding Node ID as second entry

- Now, each helper node picks up the "RSS_PACKET" with maximum Received Signal Strength(RSS) value and stores the second entry Node ID as its next hop

- Hence, every helper node chooses as its next hop the node nearest to base station out of its neighbouring helper nodes

\section{E. Sensing, Aggregation and Routing: Steady State Phase}

All above four stages form set-up phase and now, actual data transfer begins. Cluster Heads follow a TDMA schedule to assign timeslots for the sensor nodes inside the cluster. The sensor nodes send the sensed data to the corresponding Cluster Heads. The Cluster Heads aggregate the data, remove redundancies and forward the data to their Helper Nodes among which the actual Routing takes place. All other nodes except helper nodes go into sleep mode while routing takes place.

The Design of Protocol Operation is shown in Fig. 1. The data sensed at sensor nodes inside a cluster is sent to the Cluster Head. Cluster Heads Aggregate the data and send it to the Helper Node where all helper nodes are shown with links between them forming a virtual Routing Ground for routing aggregated data to the base station. Flow Chart of Operational Steps in shown in Fig. 2. Each round starts with the selection of cluster heads followed by formation of clusters by assigning cluster heads to all nodes in the network. This is followed by Helpers Nodes Selection and Route Set-Up between the Helpers Nodes by assigning next hop helper at each of the helper nodes to reach base station. This is followed by actual sensing, data aggregation and routing the aggregated data to base station.

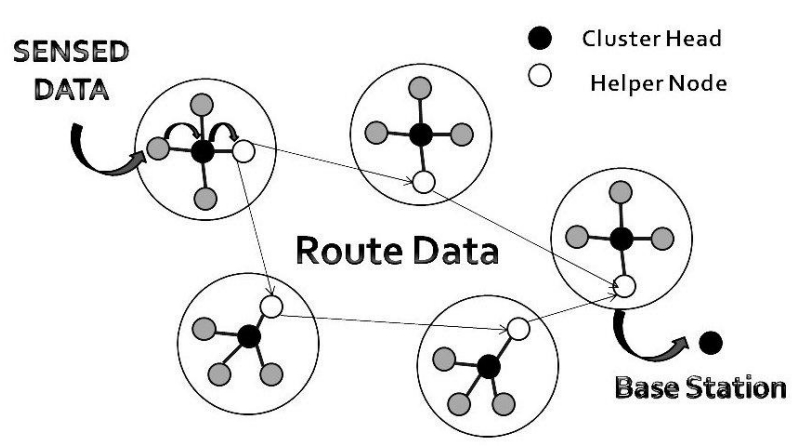

Fig. 1. Design for protocol operation.

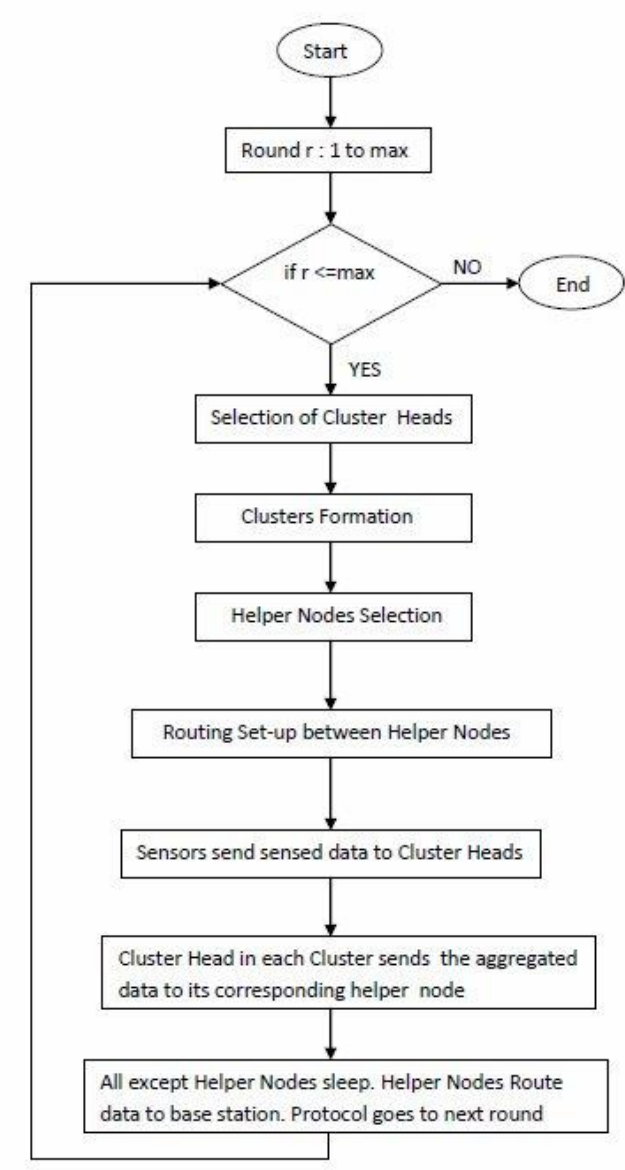

Fig. 2. Flow chart for protocol operation.

\section{ENERGY MODEL FOR COMMUNICATION}

We assume a simple model accounting for the energy dissipation in routing packets inside the network similar to most of the clustering protocols [8].

$$
\begin{aligned}
& =k \cdot E_{\text {elec }}+k \cdot \epsilon_{\text {amp }} \cdot d^{2} \quad d \leq d^{0} \\
& =k \cdot E_{\text {elec }}+k \cdot \epsilon_{\text {amp }} \cdot d^{4} \quad d>d^{0}
\end{aligned}
$$

The dissipation energy for receiver $=k \cdot E_{\text {elec }}$

where

$E_{\text {elec }}$ : Energy dissipated to run transmitter radio electronics 
$\epsilon_{a m p:}$ Energy dissipation of transmission amplifier

$k$ : Message length in bits

$d$ : Distance between nodes

$d^{0}$ : Threshold distance

The threshold distance $d^{0}$ in the Simulations is assumed to be 70 percent of the maximum possible distance between the nodes in network during our simulations. Sensors transmit data to cluster head only when an event occurs. The base station node is assumed to be a high energy node or rechargeable node facilitating beckoning to all nodes during protocol operation.

\section{THEORETICAL ANALYSIS}

LEACH [2] uses Single Hop transmission from Cluster Heads to Sink. Earlier Studies [9] show that Multi-hop relay from Nodes to Base Station is Energy Efficient than Single Hop Relay in most of the cases. We show here that Multi-hop Relay between Helper Nodes increases network lifetime compared to Multi-Hop between Cluster Heads [4] by reducing overall load of clustering and routing upon Cluster Heads.

Here, we compare the worst case energy dissipations for multi-hop transmission between Helper Nodes vs multi-hop transmission between Cluster Heads.

\section{A. Multi-Hop via Cluster Heads}

In worst case, the nearest possibility of a node to be cluster head again after once is $\left\lfloor\frac{1}{P}\right\rfloor$ rounds later $(P$ - cluster percentage). Consider two rounds ' $r$ ' and ' $r+\left\lfloor\frac{1}{P}\right\rfloor$ ' with two particular nodes $\left(\mathrm{CH}_{1}, \mathrm{CH}_{2}\right)$ from two different clusters which are cluster heads in both rounds.

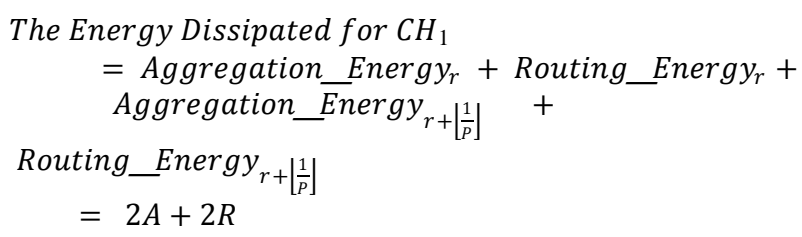

(Assume both nodes have shown up same Aggregation energy ' $A$ ' and Routing Energy ' $R$ ')

The Energy Dissipated for $\mathrm{CH}_{2}$

$$
\begin{aligned}
= & \text { Aggregation_Energy } y_{r}+\text { Routing_Energy }_{r}+ \\
& \text { Aggregation_Energy }{ }_{r+\left\lfloor\frac{1}{p}\right\rfloor}+\text { Routing_Energy }_{r+\left\lfloor\frac{1}{p}\right\rfloor} \\
= & 2 A+2 R
\end{aligned}
$$

\section{(Assume both nodes have shown up same} Aggregation energy ' $A$ ' and Routing Energy ' $R$ ')

\section{B. Multi-Hop via Helper Nodes}

This is the scenario shown up in our protocol A-LEACH. Consider two rounds ' $r$ ' and ' $r+\left\lfloor\frac{1}{p}\right\rfloor$ ' with two particular nodes which can be (ClusterHead,Helper) or $\left(\right.$ Helper $_{1}$, Helper $\left._{2}\right)$ or (ClusterHead, ClusterHead $\left._{2}\right)$ Applying the fact that Cluster Heads Aggregate Data (Aggregation Dissipation Energy - A) and Helper Nodes Route (Routing Dissipation Energy - R), Energy Dissipations are notated as follows for the possible cases:

$$
\begin{aligned}
& \text { E(ClusterHead,Helper) } \\
& =2 A \text { (For Cluster Head in Both Rounds) } \\
& =2 R \text { (For Helper Node in Both Rounds) } \\
& \text { E(Helper } \left., \text { Helper }_{2}\right)
\end{aligned}
$$

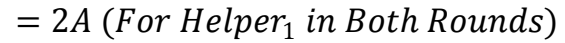

$$
\begin{aligned}
& =2 R \text { (For Helper } 2 \text { in Both Rounds) }
\end{aligned}
$$

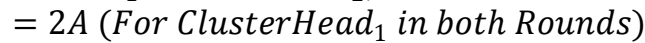

$$
\begin{aligned}
& =2 R \text { (For ClusterHead } 2 \text { in both Rounds) }
\end{aligned}
$$

All the above three cases show that total energy dissipation in both rounds for each of the two considered nodes ( $2 \mathrm{R}$ or $2 \mathrm{~A})$ is less in section IV- 2 than that of individual nodes dissipation energy $(2 \mathrm{~A}+2 \mathrm{R})$ in Section IV-1.

Hence, we can say that the multi-hop routing with concept of sharing Routing and Data Aggregation between Helper Nodes and Cluster Heads increases Network Life Time.

\section{PSEUdo CODES}

\section{A. Cluster Head Selection}

Nodes $\leftarrow$ Set of all Nodes

$\forall \mathrm{n}$ in Nodes:

if(Round $\underline{H i s t o r y} \underline{\mathrm{CH}}[\mathrm{n}] \geq$

$$
\text { Th } \leftarrow \frac{\left.\left\lfloor\frac{1}{\mathrm{P}}\right\rfloor \text { \& Round } \underline{\text { History }} \underline{H N}[\mathrm{n}] \geq\left\lfloor\frac{1}{\mathrm{P}}\right\rfloor\right)}{1-\mathrm{P} *\left(\mathrm{r} \bmod \left[\frac{1}{\mathrm{P}}\right\rfloor\right)}
$$

$/ * \mathrm{n}$ is neither cluster head nor helper in last $\left\lfloor\frac{1}{\mathrm{P}}\right\rfloor$ rounds $* /$

elseif(Roundㅍistory $\underline{\mathrm{CH}}[\mathrm{n}] \geq\left\lfloor\frac{1}{\mathrm{P}}\right\rfloor$ )

Th $\leftarrow 0.5 * \frac{\mathrm{P}}{1-\mathrm{P} *\left(\mathrm{r} \bmod \left[\frac{1}{\mathrm{P}}\right]\right)}$

$/ * \mathrm{n}$ isn't cluster head in last $\left\lfloor\frac{1}{\mathrm{P}}\right\rfloor$ rounds but can be Helper $* /$

else

Th $\leftarrow 0$

endif

if(random number in $(0,1)<T h)$

state $[n] \leftarrow$ HEAD

endif

\section{B. Cluster Formation}

Nodes $\leftarrow$ Set of all Nodes

$\forall \mathrm{n}$ in Nodes:

if $($ state $[\mathrm{n}]=$ HEAD)

broadcast HEADBOAST messages with node's ID

wait for JOINCLUSTER messages

/* JOINCLUSTER is request of a node to it's Cluster Head to join it $s$ Cluster */

/* HEADBOAST is declaration that node is a head $*$ / if(receive JOINCLUSTER from node with id = "ID") ADD "ID" to clustermembers[n]

endif

endif

if(state[n] = NORMAL)

receive all HEADBOAST messages

Select the "Cluster Head" with maximum RSS

Send JOINCLUSTER messages to "Cluster Head" myhead[n] $\nleftarrow$ "Cluster Head"

endif

/* HEADBOAST is declaration that node is a head */

/*RSS is received signal strength */ 


\section{Helper Node Selection}

Nodes $\leftarrow$ Set of all Nodes

$\forall \mathrm{n}$ in Nodes:

if (state[n] = NORMAL)

receive BaseStation $\underline{B e c k o n}$

/* Beckon Signal sent from basestation to all nodes */ send a copy of RSSPACKET(RSS(BaseStation Beckon), NODE ID) to myhead[n]

/* All nodes send received signal strength of beckon from basestation to their cluster heads $*$ /

wait for HELPERBOAST from myhead[n]

if(receive HELPERBOAST from myhead[n]) state[n] $\leftarrow$ HELPER

/* HELPERBOAST is declaration of node to be helper */ endif

endif

if $($ state $[n]=H E A D)$

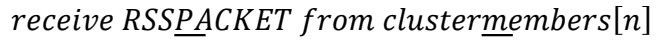
/* RSSPACKET contains RSS(BaseStationBeckon)i.e. received signal strength of beckon signal from base basestation and NODE ID*/

select "member" with highest RSS(BaseStation $\underline{\text { Beckon) }}$ send HELPERBOAST to "member" endif

/* HELPERBOAST is declaration of node to be helper */

\section{Routing Set-Up Phase to Find Next Hop in Routing}

HelperNodesList $\leftarrow$ Set of all Helper Nodes

$\forall \mathrm{n}$ in HelperNodesList:

send RSSPACKET over it's transmission range

/* To Neighbouring Helper Nodes */

/* RSSPACKET is the packet made in Helper Node selection phase (section V.3) which contains received signal strength of beckon signal sent by basestation and node id */

$\forall \mathrm{n}$ in HelperNodesList:

wait for RSSPACKET from neighbours

/* Neighbours are neighbouring helper nodes whose transmission range includes node $\mathrm{n} * /$ if(receive RSSPACKET from Neighbours) Add RSSPACKET to NextHopsList endif

Select Node with highest RSS value inside RSSPACKET from NexthopsList as "Next Hop"

\section{PERformance Results}

TABLE I: SIMULATION PARAMETERS

\begin{tabular}{|l|l|}
\hline $\mathrm{K}$ & 600 \\
\hline $\mathrm{E}_{\text {elec }}$ & $50 \mathrm{~nJ} / \mathrm{bit}$ \\
\hline$€_{\mathrm{amp}}$ & $100 \mathrm{pJ} / \mathrm{bit} / \mathrm{sq}$. unit \\
\hline Bit Compression Cost & $5 \mathrm{pJ} / \mathrm{bit}$ \\
\hline Energy of Node & $2 \mathrm{~J}$ \\
\hline Nodes Number & 100 \\
\hline Rounds & 2000 \\
\hline Area & $100 X 100$ sq.units \\
\hline Cluster Percentage & 0.1 \\
\hline
\end{tabular}

We have simulated direct transmission, multi-hop transmission, Leach and our proposed protocol Assisted Leach in $\mathrm{C}$ language. We have substantiated our theoretical analysis with simulation results using the parameters shown in Table I. We have collected the round numbers for deaths of all nodes during simulation of the four protocols. For optimal comparison, data for death of half the network is shown in Table II and Fig. 3 is the plot for the whole data. We have collected the values of total energy in the network after every death in nodes for all the four protocols. Table III has the data for an intermediate round depicting the network energies and Fig. 4 is the plot for all the data. Half of the network in other protocols dies at almost half the rounds taken for death of half the network in Assisted-Leach.

TABLE II: Round VS NODE DEATH FOR DEATH OF HALF THE NETWORK

\begin{tabular}{|c|c|c|}
\hline Protocol & Death Count & Round \\
\hline Direct & 45 & 117 \\
\hline Multi-Hop & 45 & 199 \\
\hline Leach & 45 & 443 \\
\hline Assisted-Leach & 45 & 803 \\
\hline
\end{tabular}

TABLE III: ENERGY VS ROUNDS FOR AN INTERMEDIATE ROUND

\begin{tabular}{|c|c|c|}
\hline Protocol & Round & Network Energy \\
\hline Direct & 600 & 0 \\
\hline Multi-Hop & 600 & 0 \\
\hline Leach & 600 & 59.851421 \\
\hline Assisted-Leach & 600 & 109.714081 \\
\hline
\end{tabular}

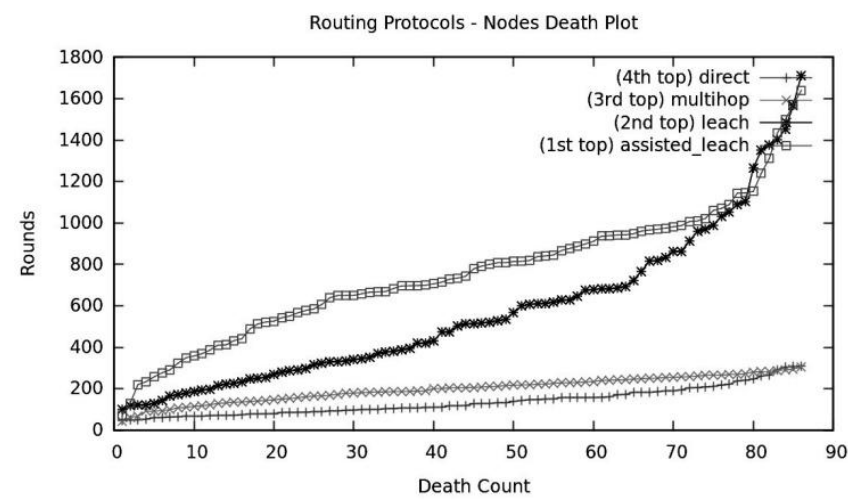

Fig. 3. Rounds vs node deaths.

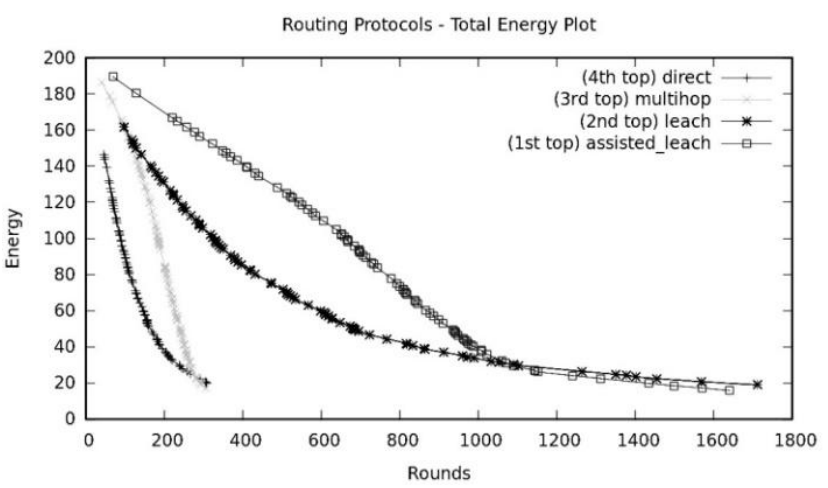

Fig. 4. Network energy vs rounds.

\section{CONCLUSION}

Our Theoretical Analysis has shown that network lifetime goes down when both data aggregation and routing are carried out by Cluster Heads alone which can be eradicated by usage of Helper Nodes for Routing and Cluster Heads for Data Aggregation. We have reduced the overhead for route formulation to base station by electing next hop at each Helper Node using the Received Signal Strength values of beckon signal from base station already available at helper nodes during Helper Node Selection phase. Our concept of 
Helper Nodes in Assisted LEACH (A-LEACH) protocol has improved the lifetime of the network by distributing the minimized energy dissipation throughout the nodes. Theoretical analysis and simulation results substantiate this.

\section{ACKNOWLEDGEMENT}

Thanks to Professor Ajit Pal and few other Professors at CSE, IIT Kharagpur for assistance in idea formulation and validating the simulation results.

\section{REFERENCES}

[1] F. Akyildiz and W. Su, "A survey on sensor networks," Communications Magazine, IEEE, vol. 40, no. 8, pp. 102-114, 2002.

[2] W. Heinzelman, A. Chandrakasan, and H. Balakrishnan, "Energy-Efficient communication protocol for wireless sensor networks," in Proc. the Hawaii International Conference on System Sciences, Hawaii, January 2000.

[3] A. Joshi and P. M. Lakshmi, "A survey of hierarchical routing protocols in wireless sensor networks," International Journal of Wireless and Mobile Networks, vol. 4, no. 4, Aug. 2012.

[4] R. V. Biradar, S. R. Sawant, R. R. Mudholkar, and V. C. Patil, "Multihop routing in self-organizing wireless sensor networks," in Proc. IJCSI International Journal of Computer Science Issues, vol. 8, issue 1, January 2011, pp. 155-164.

[5] J. F. Yan and Y. L. Liu, "Improved leach routing protocol for large scale wireless sensor networks routing," in Proc. 2011 International Conference on Electronics, Communications and Control (ICECC), pp. 3754-3757.

[6] M. O. Faroog, A. B. Dogar, and G. A. Shah, "MR-LEACH: Multi-hop routing with low energy adaptive clustering hierarchy," in Proc. 2010 Fourth International Conference on Sensor Technologies and Applications, pp. 262-268.

[7] J. Wang, G. Yang, and S. Chen, "Secure LEACH routing protocol based on low-power cluster-head selection algorithm for wireless sensor networks," in Proc. 2007 International Symposium on Intelligent Signal Processing and Communication Systems, Nov.28-Dec.1, 2007 Xiamen, China, pp. 341-344.

[8] E. Abdellah, S. Benalla, A. B. Hssane, and M. L. Hasnaoui, "Advanced low energy adaptive clustering hierarchy," International Journal on Computer Science and Engineering, vol. 02, no. 07, pp. 2491-2497, 2010.

[9] S. Fedor and M. Collier, "On the problem of energy efficiency of multi-hop vs one-hop routing in wireless sensor networks," in Proc. 21 th International Conference on Advanced Information Networking and Applications Workshops (AINAW'07).

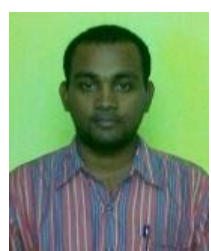

Sunkara Vinodh Kumar was born in Visakhapatnam, Andhra Pradesh on April 10, 1991. He is currently pursuing Fourth Year B. Tech (Honors) from computer science and engineering department, IIT Kharagpur, India. He was SUMMER INTERN at Telecommunication Networks Group, TU Berlin, Germany in the year 2012. He was an Indian Academy of Sciences, Bangalore SUMMER FELLOW in the year 2011. He is placed as Software Engineer at Yahoo, India during campus placements in IIT Kharagpur for the year 2013. He is first author of Technical Report published at TKN Technical Report Series, TKN-12-003, Telecommunication Networks Group, TU Berlin, 2012. He is first author of" Demo Abstract: TWIST Actu-A RESTful Testbed Platform for Remote Experimentation With Building Automation Sensors and Actuators" published at ACM BuildSys 2012 workshop, part of Sensys'12, Toronto, Canada. His Research Interests include Computer Networks, Wireless Sensor Networks. Mr. Kumar has been a Winner at Hack U, 2013 conducted by YAHOO company in IIT Kharagpur, India.

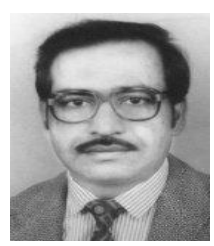

Ajit Pal was born in a village Thakdala on Oct 29, 1949. He obtained his M.Tech and Ph.D. degrees from the Institute of Radio Physics and Electronics, Calcutta University in 1971 and 1976, respectively. $\mathrm{He}$ is presently Professor, Department of Computer Science and Engineering at the Indian Institute of Technology, Kharagpur. Before joining IIT Kharagpur in the year 1982, he was with the Indian Statistical Institute, Calcutta, ITI, Naini and DLRL, Hyderabad in various capacities. He became full Professor in 1988 and served as head of Computer Center from 1993 to 1995 and head of the Computer Science and Engineering Department from 1995 to 1998. He has over 150 publications in reputed journals and conference proceedings and two book entitled"Microprocessors: Principles and Applications" published by TMH 2004 and "Microcontrollers: Principles and Applications" published by PHI 2011. His research interests include Real Time Systems, CAD for VLSI and Computer Networks. Prof $\mathrm{Pal}$ is the Fellow of the IETE, India and Senior Member of the IEEE, USA $\mathrm{He}$ is the principal investigator of several Sponsored Research Projects including" Low Power Circuits and Systems" sponsored by Intel, USA. He received 'Meghnad Saha Memorial Award - 1977' from IETE (India), 'Meghnad Saha Memorial Award - 1986' from IETE (India), Best Paper Award for the paper entitled 'Fault Tolerant Scheduling in Distributed Real-Time Systems', Beizing, China, Oct 2001. 